\title{
Postoperative Bleeding and Leakage After Sleeve Gastrectomy: a Single-Center Experience
}

\author{
Mousa Khoursheed $^{1}$ - Ibtisam Al-Bader ${ }^{1}$ - Ali Mouzannar ${ }^{2} \cdot$ Aqeel Ashraf $^{2}$. \\ Yousef Bahzad $^{2}$ • Abdulla Al-Haddad ${ }^{2}$ - Ali Sayed ${ }^{2} \cdot$ Abe Fingerhut $^{3}$
}

Published online: 11 October 2016

(C) Springer Science+Business Media New York 2016

Postoperative bleeding was defined as a drop of hemoglobin of more than $2 \mathrm{~g}$ associated with pallor, tachycardia, and abdominal pain and distension. Intra-abdominal fluid collections were sought by abdominal CT scan.

All patients who had bleeding (as defined before) required blood transfusion; however, major bleeding that required reoperation was seen in three patients who presented not only with drops of hemoglobin and tachycardia but they were in a state of shock, and they required urgent resuscitation and reoperation laparoscopically. All patients presented with postoperative bleeding and none was intraoperative [1].

Our rate of postoperative bleeding was within the range of what has been reported in the literature, and we did not encounter intraoperative bleeding.

We avoid using clips or electrocoagulation since we think they are unsafe and may contribute to increase risk of postoperative leak. Instead, we suture the staple line, which is cheap too and we inspect it carefully. If we notice any further bleeding, we reinforce the site of bleeding with figure of eight stitch rather than using clips or electrocoagulation. With this technique that we have adopted lately, we have noticed further reduction in our postoperative bleeding rate.

We had no leaks in our series and regional ischemia, and leak following oversewing was shown in animal studies and was not supported by clinical studies.

Mousa Khoursheed

khoursheed@hsc.edu.kw

\footnotetext{
Kuwait University, Kuwait, Kuwait

Mubarak Al-Kabeer Hospital, Jabriya, Kuwait

3 Medical University of Graz, Graz, Austria
}

The aim of our study was to look at two early major complications after sleeve gastrectomy and not a systematic collection of patient-reported outcomes such as physical activity, diet, and quality of life.

\section{Compliance with Ethical Standards}

Conflict of Interest The authors declare that they have no conflicts of interests.

Ethical Approval All procedures performed in studies involving human participants were in accordance with the ethical standards of the institutional and/or national research committee and with the 1964 Helsinki declaration and its later amendments or comparable ethical standards.

Informed Consent For this type of study, formal consent is not required.

\section{References}

1. Khoursheed M, Al-Bader I, Mouzannar A, Ashraf A, Bahzad Y, AlHaddad A, Sayed A, Fingerhut A. Postoperative bleeding and leakage after sleeve gastrectomy: a single-center experience. Obes Surg. 2016. 